\title{
Using Social Network Analysis to Identify Spatiotemporal Spread Patterns of COVID-19 around the World: Online Dashboard Development
}

\author{
Kyent-Yon Yie ${ }^{1}$, Tsair-Wei Chien ${ }^{2}$ D, Yu-Tsen Yeh $^{3}$, Willy Chou ${ }^{4, *}$ and Shih-Bin Su ${ }^{5, *(D)}$ \\ 1 Department of Gastrointestinal Hepatobiliary, Chi Mei Jiali Hospital, Tainan 700, Taiwan; \\ kyawsein@gnail.com \\ 2 Department of Medical Research, Chi-Mei Hospital, Tainan 700, Taiwan; smile@mail.chimei.org.tw \\ 3 Medical School, St. George's University of London, London SW17 0RE, UK; jess97yeh@gmail.com \\ 4 Department of Physical Medicine and Rehabilitation, Chi Mei Medical Center, Tainan 700, Taiwan \\ 5 Department of Occupational Medicine, Chi Mei Medical Center, Tainan 700, Taiwan \\ * Correspondence: ufan0101@ms22.hinet.net (W.C.); shihbin1029@gmail.com (S.-B.S.); \\ Tel.: +886-6291-2811 (S.-B.S.)
}

\section{check for}

updates

Citation: Yie, K.-Y.; Chien, T.-W.; Yeh, Y.-T.; Chou, W.; Su, S.-B. Using Social Network Analysis to Identify Spatiotemporal Spread Patterns of COVID-19 around the World: Online Dashboard Development. Int. J. Environ. Res. Public Health 2021, 18 , 2461. https://doi.org/10.3390/ ijerph18052461

Academic Editors: Joan Puig-Barberà and George Kassianos

Received: 29 January 2021

Accepted: 25 February 2021

Published: 3 March 2021

Publisher's Note: MDPI stays neutral with regard to jurisdictional claims in published maps and institutional affiliations.

Copyright: (c) 2021 by the authors. Licensee MDPI, Basel, Switzerland. This article is an open access article distributed under the terms and conditions of the Creative Commons Attribution (CC BY) license (https:// creativecommons.org/licenses/by/ $4.0 /)$.

\begin{abstract}
The COVID-19 pandemic has spread widely around the world. Many mathematical models have been proposed to investigate the inflection point (IP) and the spread pattern of COVID-19. However, no researchers have applied social network analysis (SNA) to cluster their characteristics. We aimed to illustrate the use of SNA to identify the spread clusters of COVID-19. Cumulative numbers of infected cases (CNICs) in countries/regions were downloaded from GitHub. The CNIC patterns were extracted from SNA based on CNICs between countries/regions. The item response model (IRT) was applied to create a general predictive model for each country/region. The IP days were obtained from the IRT model. The location parameters in continents, China, and the United States were compared. The results showed that (1) three clusters (255, $\mathrm{n}=51,130$, and 74 in patterns from Eastern Asia and Europe to America) were separated using SNA, (2) China had a shorter mean IP and smaller mean location parameter than other counterparts, and (3) an online dashboard was used to display the clusters along with IP days for each country/region. Spatiotemporal spread patterns can be clustered using SNA and correlation coefficients (CCs). A dashboard with spread clusters and IP days is recommended to epidemiologists and researchers and is not limited to the COVID-19 pandemic.
\end{abstract}

Keywords: COVID-19; social network analysis; item response model; correlation coefficient; daily confirmed case; spatiotemporal spread pattern

\section{Introduction}

The COVID-19 pandemic is spreading widely around the world, causing significant threats to the public. As of February 20, the world has accumulated more than 0.11 billion the COVID-19 cases, threatening people's health, economic development, and social stability [1]. Many online real- or near-real-time dashboards have been launched to track the worldwide spread of the COVID-19 outbreak [2] and to provide the cumulative numbers of infected cases (CNIC) daily to the public, such as the Johns Hopkins University Center for Systems Science and Engineering dashboard (JHU) [3], the Leszkiewicz personal dashboard [4], the World Health Organization dashboard [5], HealthMap [6], and the dashboard created by Schiffmann, an 18-year-old high school senior from Washington State in the United States [7].

Although those website owners [3-7] made efforts to launch dashboards, the sites merely display the basic information on the CNIC of COVID-19 with bubbles on a world map. Other websites [8-12] also provide common and regular information (e.g., with a traditional world map and CNIC or daily confirmed cases (DCC)) to the public. None of 
these websites are equipped with spatiotemporal spread patterns (SSP) of COVID-19 and the inflection points (IP) [13-16] to present the negative impact of COVID-19 (NISHC) on a world map using an overall score to fulfill the public's interest. Displaying CNIC bubbles on world maps in areas is common during the COVID-19 pandemic. Determining how to display both features (i.e., SSP and IP days) on a world map is challenging. We were thus motivated to design a dashboard that can simultaneously display both SSP and IP days and complementarily characterize spread patterns of COVID-19 across the globe.

Furthermore, few authors are willing to provide their detailed experimental data to readers who are going to replicate the study on their own. Data, along with MP4 videos and modules in articles, would be an interesting resource and have rarely been seen before in the literature.

\subsection{Social Network Analysis for Identifying Spread Patterns Based on DCC}

Social network analysis (SNA) [17] is the process of investigating social structures through the use of networks and graph theory. It characterizes networked structures in terms of nodes (individuals, groups of people, or objects within the network) and the ties, edges, or links (relationships or interactions) that connect them. Examples of social structures commonly visualized through SNA can be found in many academic disciplines and fields [18-23]. The use of SNA was thus expected to identify SSP of the COVID-19 cases around the world.

\subsection{Item Response Theory and the Infection Point on an Ogive Curve}

Many methods [24-32] have been used to build mathematical models for predicting the CNIC or DCC of COVID-19. None of the previous studies applied the item response theory (IRT) $[33,34]$ to the pandemic outbreak. The IRT model, using probability to display the ogive curve (based on CNIC), is emulated as the epidemic trend on the expected CNCI. Two parameters (i.e., location on the $x$-axis and slope as the steep of a curve toward the $y$-axis represented by item difficulty (b) and discrimination (a), respectively) are involved in a predictive model. The infected days on the $x$-axis are transformed to be the so-called ability parameter $(\theta)$ on a continuum scale from the left to the right side (e.g., in a range from -5 to 5) [32]. As such, the cumulative probabilities on the $y$-axis can be converted back to the expected CNIC for a specific country/region that has been impacted by COVID-19. The inflection point (IP) [13-16,32] can be determined on the IRT ogive curve accordingly.

\subsection{Two Phenomena Observed in the COVID-19 Pandemic}

We observed that the SARS-CoV-2 (COVID-19) disease began in Wuhan, China [35] and was spread to West Asia, Europe, North America, and South America. Whether the spread routes of COVID-19 are evident using SNA is worth studying.

After entering the winter season, starting in January, the deadly coronavirus experienced a new wave (called the second wave) of sporadic cluster cases, especially in colder cities. For example, the northern and northeastern cities in China, including Shijiazhuang City in Hebei Province and Suihua City in Heilongjiang Province, began to report new cluster cases [36]. Shijiazhuang City in Hebei Province has been listed as a high-risk region. Currently, Shijiazhuang City is the only high-risk region in China, with over 300 new confirmed cases, a record high in over 5 months, in the 10 days from 2 to 11 January 2021 [36]. Two questions were raised: (1) Whether both the provinces of Hebei and Heilongiiang in China have a similar spread pattern, and (2) which countries (or regions) have the same spread pattern as Hebei Province (China).

\subsection{The Aims of This Study}

Two parts are involved in this study: Identifying the spread patterns in the two periods (1) from 22 January to 27 March 2020, and (2) from 1 January to 16 February 2021. The aims of this study are to (1) illustrate the use of SNA for investigating the association of DCCs in regions; (2) examine the spread routes of COVID-19 from China to West Asia, Europe, 
North America, and South America; (3) identify countries/regions with the same spread pattern as Hebei Province in China; and (4) design a dashboard for better interpretation of CNIC and the corresponding IP days by country/region.

\section{Materials and Methods}

\subsection{Data Source}

We downloaded COVID-19 outbreak DCCs in the two periods (1) from 22 January to 27 March 2020, and (2) from 1 January to 16 February 2021, as mentioned above from GitHub [8], a site that provides information on newly DCCs in countries/regions around the world. All the downloaded data with 299 countries/regions are publicly displayed on the website. Ethical approval is not necessary for this study because all the data are obtained via the Internet (see Appendix A).

\subsection{Spread Routes of COVID-19 across Continents}

Although we knew that the spread routes of COVID-19 were from Asia to Europe and then North and South America, the spread routes are still unknown. In March 2020, the US Centers for Disease Control and Prevention (CDC) established geographic risk stratification criteria for the purpose of issuing travel health notices for countries with COVID-19 risk and guiding management decisions for people with potential travel exposures to COVID19 [37]. The restriction on entry to the United States for foreign nationals from China and Iran was issued on 16 March 2020 or earlier. The lag-behind spread pattern is required for investigations on a different DCC pattern for COVID-19 between China and the US. The spread routes are thus tracked with SSP using SNA.

\subsection{The Three Steps Below Were to Identify the Spread Patterns of COVID-19}

Step 1: Using log (CNIC) to define the correlation coefficients (CCs) in countries/regions

The sequential $\log$ (CNICs) in each region were extracted from the data [8] to compare the pattern with the CCs between countries/regions (see the MP4 video in Appendix A). For instance, we selected the most similar CNIC patterns (i.e., with higher CCs) for Hubei (China) in Figure 1. It can be seen that Heilongjiang (China), Xinjiang (China), and other regions in China were highlighted in Stage I (e.g., in the first one-third of days during the study period up to 27 March 2020). However, other regions were shown in Stage II (e.g., Guangdong (China) and Guangxi (China)) and Stage III (e.g., Egypt, Iran, Sweden, the Republic of Korea, and Gansu (China)), all of which were dated 27 March 2020. As such, different spread patterns can be examined using the CCs on CNIC across countries/regions.

Step 2: Applying SNA to classify the spread clusters of COVID-19

The CCs for each paired country were included in SNA (see the control file of Pajeck [38] in Appendix A). The higher CCs that a region was associated with, the closer the relationship was within a cluster for regions with identical CNIC patterns.

In keeping with Pajek's guidelines [38] for SNA, we defined a region as a node (or an actor/vertex) that is connected to another node through an edge (or tie with a line) (i.e., the weight denoted by CC on CNIC between 2 entities). Usually, the weight (dented by $\mathrm{CC}$ ) is summed (called concentrality degree (CD) by the number of connections between 2 nodes). The more connections (or said co-occurrence) produce a higher $\mathrm{CD}$ in the network, the algorithm of the community partitioning method was used to identify and separate clusters in SNA. The number of clusters was set at 3 in the SNA based on Pajeck's criteria. The SNA file is provided in Appendix A as well.

Step 3: Plotting the SNA to classify the spread clusters of COVID-19

A dashboard was designed for displaying region bubbles colored by cluster types and sized by the weights (i.e., $\mathrm{CD}$ denoted by the summation of CCs) on Google Maps. The line (or curve) linked to the 2 nodes stands for the closer relation (or CC $>0.8$ or more). The curve disappears if the CC is less than the criterion of 0.8 . 
Two types of SNA were drawn according to the research design (i.e., one for data from 22 January to 27 March 2020, and another from 1 January to 16 February 2021), including (1) the spread routes in stages, and (2) the SSP of other countries/regions related to Hebei Province (China) and Heilongjiang (China).

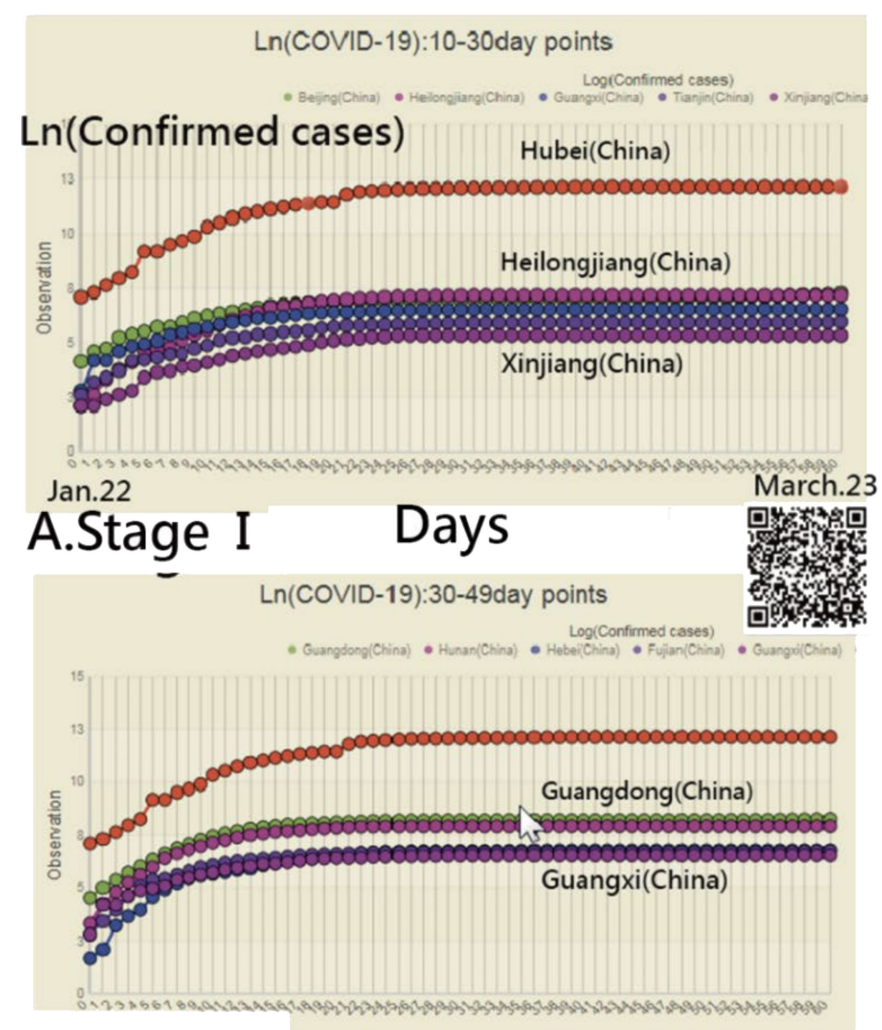

\section{B.Stage II Days}

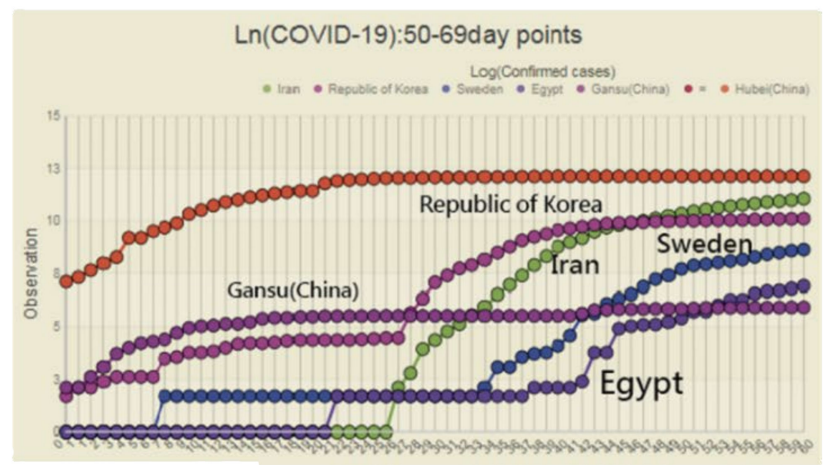

\section{C.Stage III Days}

Figure 1. Matching the most similar regions using the correlation pattern across three clusters (stages) (Note: readers are invited to scan the QR-code to examine the detail on a dashboard).

\subsection{Building the Model-Based on IRT}

\subsubsection{Percentage-Type Observed Data (OPi)}

The CNIC in a country/region was transformed into a percentage from 0 to 1 [39-41], as shown in Equation (1).

$$
\mathrm{OPi}=\frac{(\mathrm{Oi}-\mathrm{Min})}{(\mathrm{Max}-\mathrm{Min})} \times \mathrm{CRi},
$$

where Oi denotes the originally observed case number, and the maximum and minimum are symbolled by Max and Min, respectively. The CR stands for the adjustment coefficient 
because not all TSCCC equal 1.0 at $\theta(=5)$ according to Equation (2) (e.g., at early epidemic stage [32]).

\subsubsection{The IRT Probability Model}

Two parameters, $a$ and $b$, mentioned in Section 1.2, were estimated using the IRT model in Equation (2), in which a and b (named delta) represent the discrimination (i.e., the slope shape) and the item difficulty (i.e., the location toward the left (easier or at an earlier stage) or the right (harder or at the later stage in epidemic)) with values within 0 and 3 , and -5 and 5 , respectively. Theta was set at 5 at the modeling process and used to compute the adjustment coefficient of CRi in Equation (1).

$$
\mathrm{P}(\theta)=\frac{\exp ((a(\theta-\delta))}{(1+\exp (a(\theta-\delta))}
$$

\subsubsection{Ogive Curved in a Model}

After model parameters were estimated in Equation (2), the Theta denoted by the location on the scale (Axis X) was determined by Equation (3).

$$
\text { Theta }=-5+\left(\mathrm{n}_{i-1}\right) \times \frac{(5-(-5))}{N},
$$

where $N$ is the observed days, and $\mathrm{n}_{i}$ represents $i$ th day. The probability (or denoted by the expected percentage, Epi) can be yielded by Equation (2). For instance, the Theta on the day $i$ in a total $N$ days in epidemic can be obtained by Equation (3).

\subsubsection{Transforming Epi into the Number of Expected CNIC}

Based on Equation (1), we can obtain the expected CNIC via Equation (4):

$$
\text { Expected } \mathrm{CNIC}=\mathrm{EPi} \div \mathrm{C} R_{i} \times(\mathrm{Max}-\mathrm{Min})+\mathrm{Min} .
$$

\subsection{Model Parameter Estimation}

\subsubsection{The Attributes of the Ogive Curve}

The shorter epidemic drives the ogive curve toward the left. Otherwise, the longer epidemic drives the curve toward the right side. The length of the IP days affects the NISHC in a specific country/region.

\subsubsection{Parameter Estimation}

We applied the Microsoft Solver add-in tool to estimate parameters (see the MP4 video in Appendix A).

A. To minimize the total residuals, we used the Microsoft function as shown below.

$$
\text { SUMXMY2 }([\mathrm{OPi}-\mathrm{Epi}] \times[\mathrm{OPi}-\mathrm{Epi}])=\sum_{i=1}^{n}(\mathrm{OPi}-\mathrm{Epi})^{2} .
$$

B. Estimated parameters In Equation (2), a and $b$ were estimated.

C. Constrained terms We set $\mathrm{a}$ and $\mathrm{b}$ in a range between $(0,4)$ and $(-5,5)$, respectively. In addition, the correlation coefficient (CC) between OPi and Epi was set beyond 0.9 .

D. Perform the Solver add-in The Microsoft Solver add-in was performed for each country/region to estimate the model parameters (see Appendix A for more details). The ogive curve can be plotted to predict the future CNIC and determine IP days as explained in the next section. 


\subsection{Determining IP Using a Search Scheme}

The IP was determined by the computation of the absolute advantage coefficient (AAC), or the dimension coefficient (DC) [32,39-41], in Equation (6).

$$
\mathrm{AAC}=\frac{\frac{\gamma_{3}}{\gamma_{2}}}{\frac{\gamma_{2}}{\gamma_{1}}},
$$

where AAC is determined by the 3 consecutive Epi, and the IP is located at the minimum one across all possible AACs on an ogive curve [32].

\subsection{Statistical Tools and Data Analysis}

A visual representation displaying the comparison of model parameters among continents/regions was plotted on the Kano diagram [42-44]. The IRT-modeling process was executed in Microsoft Excel (see Appendix A). The study flowchart is present in Figure 2.

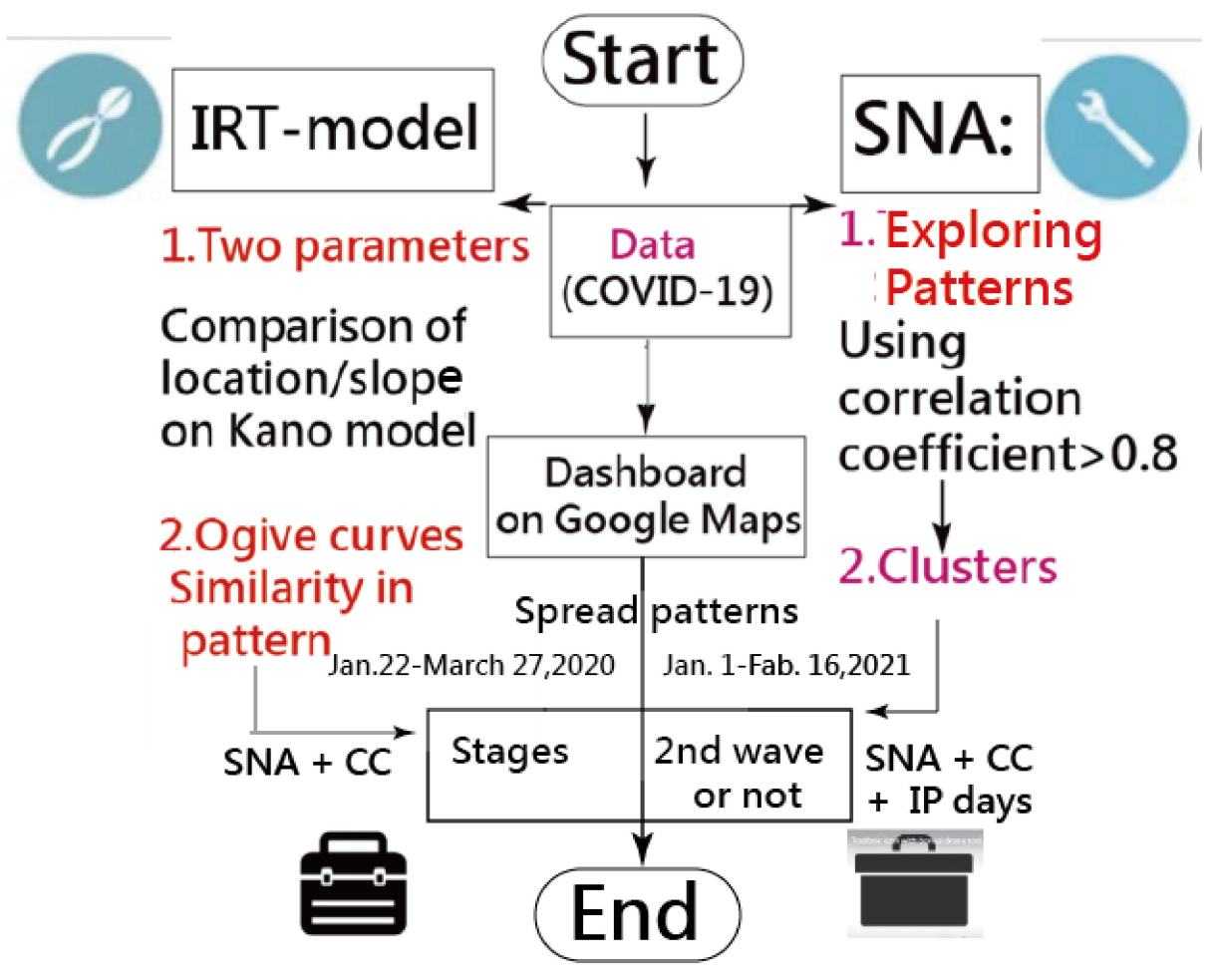

Figure 2. Study flowchart.

\section{Results}

\subsection{Spread Clusters in Color on Google Maps}

Three clusters colored by routes and stages are presented in Figure 3. We can see that the spread stages were from Asia to Europe and then to the American continent, as shown by the yellow, green, and red bubbles. Nonetheless, a few bubbles in yellow are located in Canada (North America) and Finland (Europe). Many red bubbles are present across Europe and South America. It is worth noting that Indonesia is red with curved lines (i.e., connected to other associated regions in red) at the middle bottom of Figure 3, which tells us that Indonesia had the same CNIC pattern as the US. Similarly, many red bubbles (e.g., the UK, Ireland, Norway, and Brazil) also had the same CNIC pattern as the US. Readers are invited to scan the $\mathrm{QR}$ code in Figure 3 and click on the bubble of interest to inspect the details about regions with similar COVID-19 CNIC patterns, such as those shown in Figure 1. 


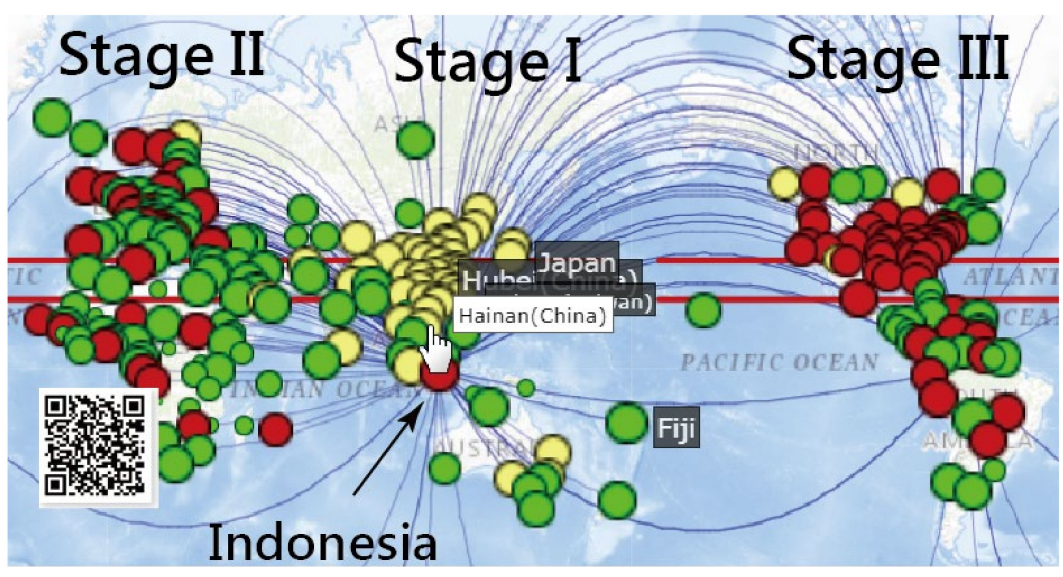

Figure 3. Indonesia compared to three clusters separated by social network analysis (SNA) using the correlation pattern across three stages in yellow, green, and red, respectively. (Note: the curve means the close relation (correlation coefficient $(C C)>0.8$ ) between two countries/regions according to SNA guidelines (Note: readers are invited to scan the QR-code to examine the detail on a dashboard)).

\subsection{Details about Indonesia in the CNIC Pattern of COVID-19}

We inspected the CNIC patterns in Indonesia from three perspectives at days 49-51, 51-53, and 54-56 (i.e., three stages) in Figure 4. We can see that many CNIC patterns in the US coincided with those in Indonesia, using the closer CC for identification.

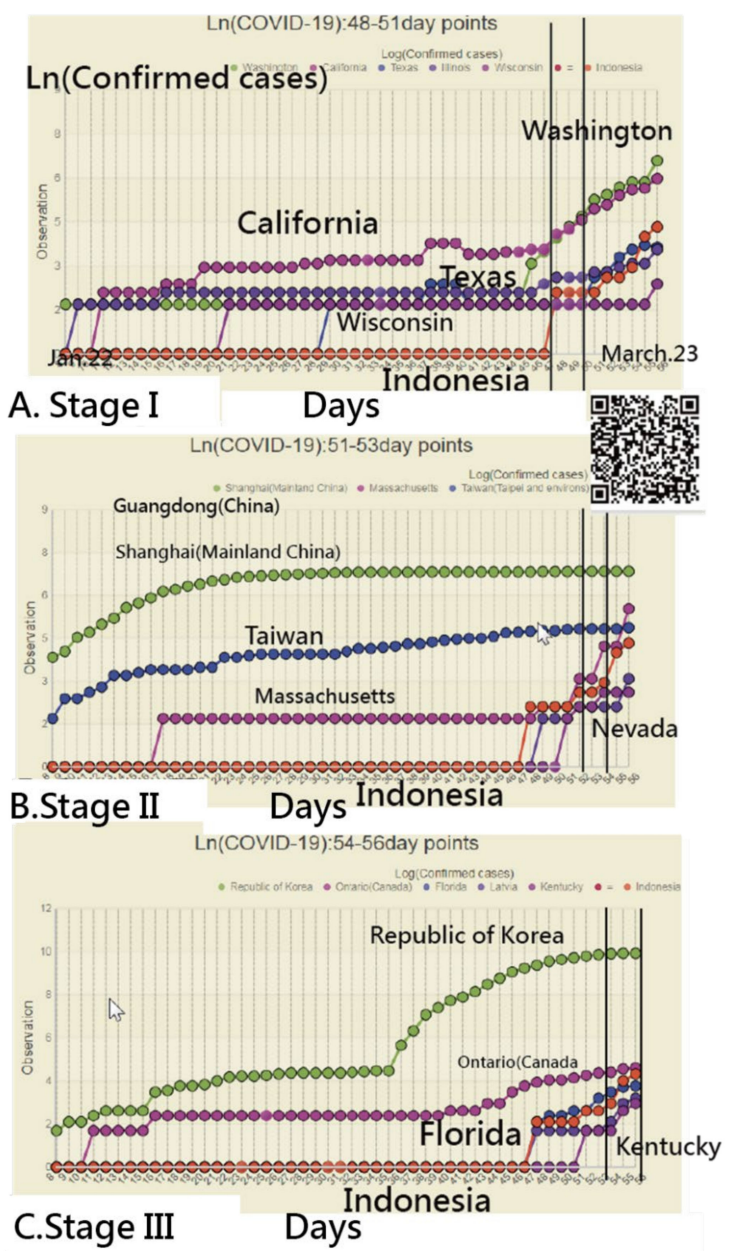

Figure 4. Using the example of Indonesia to interpret the similarities between regions across three stages (Note: readers are invited to scan the QR-code to examine the detail on a dashboard). 


\subsection{Finland's CNIC Pattern of COVID-19}

The CNIC pattern for Finland is presented in Figure 5. We chose Finland because we were curious why just one yellow bubble appeared in Europe. We can see that the CNIC pattern for Finland was much closer to those in yellow areas (e.g., regions in China) and those in green bubbles (e.g., regions in Austria and South America). It is worth noting that a few red bubbles are connected to Finland.

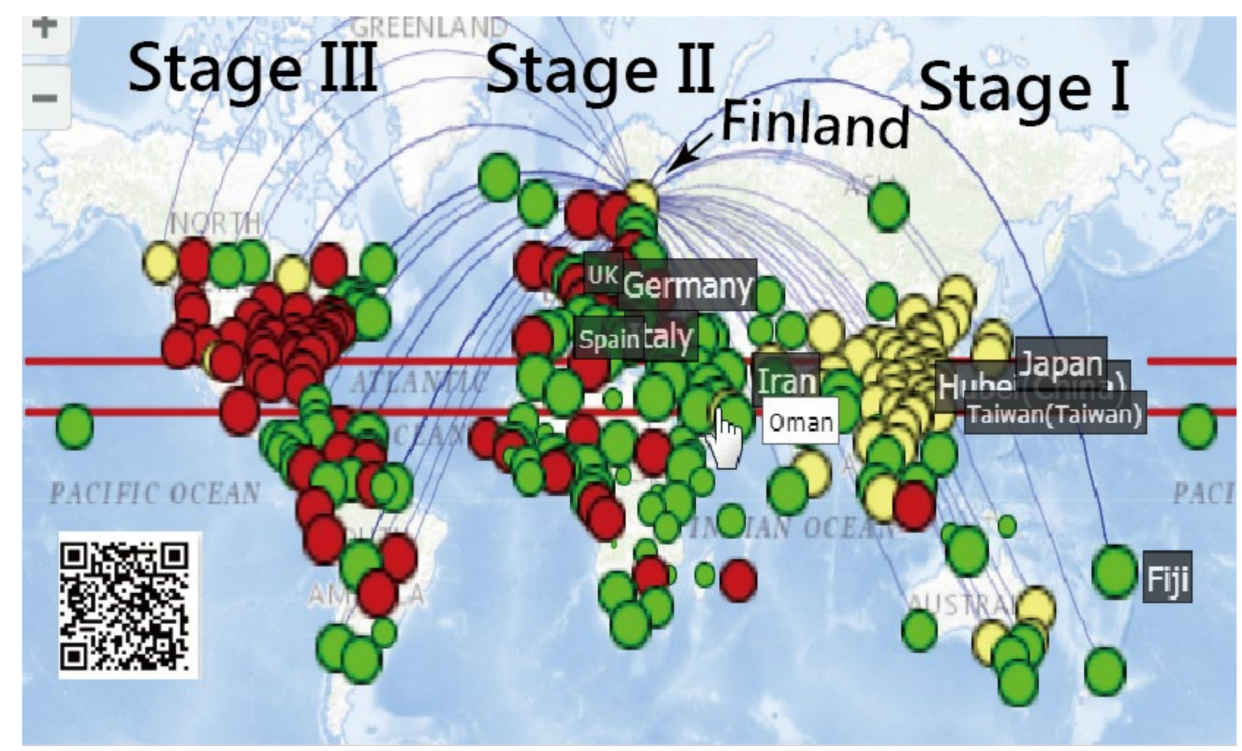

Figure 5. Finland compared to three clusters separated by SNA using the correlation pattern across three stages in yellow, green, and red, respectively. (Note: The curve means the close relation $(C C>0.8)$ between two countries/regions according to SNA guidelines; readers are invited to scan the QR-code to examine the detail on a dashboard).

\subsection{Using an IRT-Based Model to Examine Spread Patterns}

Two parameters (i.e., location $b$ and slope a) in an IRT-based COVID-19 model are present in Figure 6, in which bubbles are colored by continents and sized by the number of IP days. Three parts are separated on the Kano diagram, including a higher slope at the top, which is located at the far-right side and neutral in the middle. We can see that COVID-19 spreads from Asia (in yellow on the left) to Oceania (in brown) and Europe (in red), then to North America (in light green), Africa (in darker green), and South America (in black). Bubbles with similar patterns in the CNIC are closer together in Figure 6. For example, in Figure 7, Indonesia is similar to the US states of Wisconsin and Missouri. The observed CNICs are in black, while the predicted CNCIs are in green on the ogive curves. Readers are invited to click on the link [45]. After the bubble of interest is selected, the IRT-based ogive curve, like that in Figure 7, immediately appears on the website.

The comparison of location parameters in continents/countries as of 19 October 2020 is displayed in Table 1. We can see that the means of location parameters were lower China and higher in the US and South America. A significant difference in mean locations on Axis X in Figure 6 was found between China and the US. 


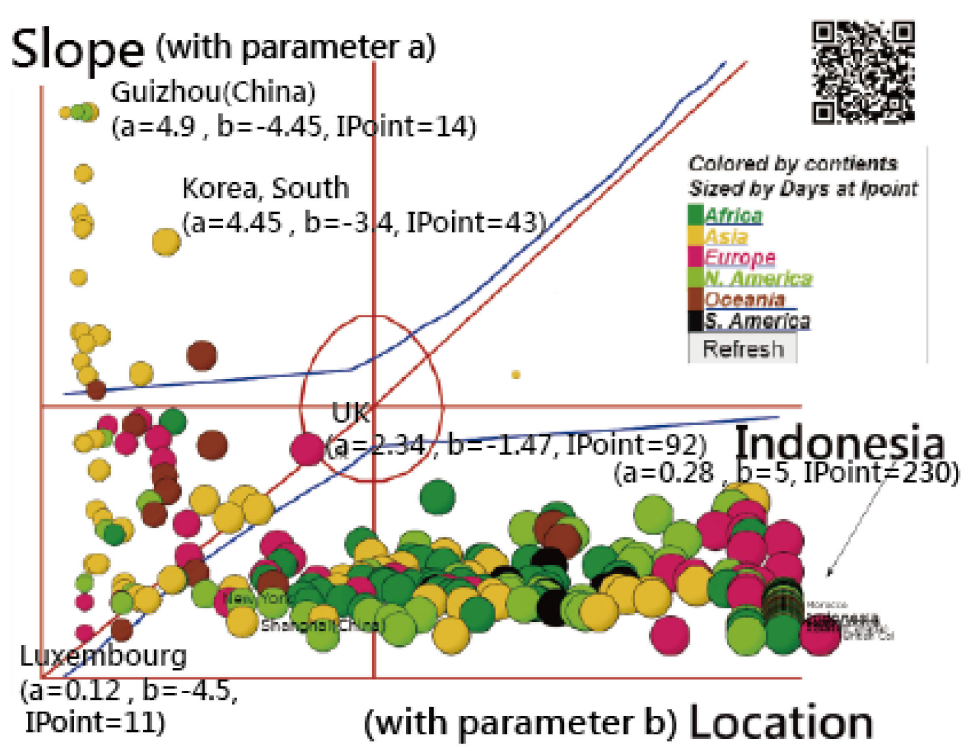

Figure 6. Similarity of the epidemic trend using the item response model (IRT) and Kano models to display the results (Note: readers are invited to scan the QR-code to examine the detail on a dashboard).

\section{Infected case}

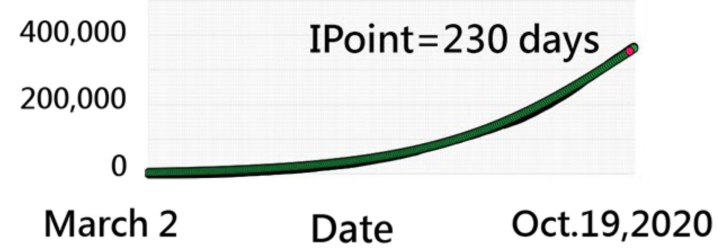

\section{A. Indonesia(ASIA)}

\section{Infected case}

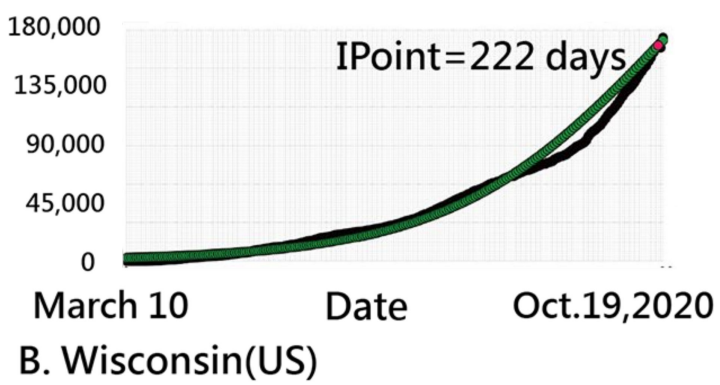

\section{Infected case}

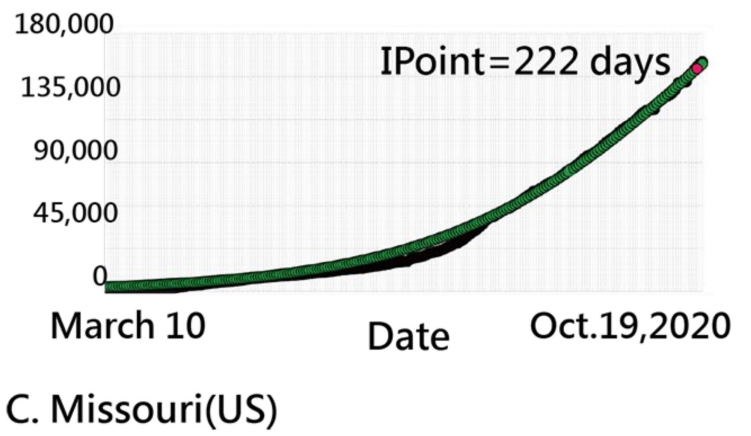

Figure 7. Comparison of epidemic trends displayed as ogive curves. 
Table 1. Comparison of location parameters in continents and countries as of 19 October 2020.

\begin{tabular}{|c|c|c|c|c|c|}
\hline \multirow{2}{*}{ Area } & \multirow{2}{*}{$n$} & \multirow{2}{*}{ Mean } & \multirow{2}{*}{ SD } & Different $(p<0.05)$ & \multirow[t]{2}{*}{ Stage } \\
\hline & & & & From Area i & \\
\hline (3) CHINA & 31 & -2.5382 & 3.1201 & $(1)(2)(4)(5)(7)(8)$ & I \\
\hline (6) OCEANIA & 15 & -1.6913 & 2.4752 & $(2)(4)(5)(7)(8)$ & \\
\hline (1) AFRICA & 53 & 1.0080 & 2.3828 & $(3)(4)$ & II \\
\hline (2) ASIA & 44 & 1.7731 & 3.1458 & $(3)(4)(6)$ & \\
\hline (5) N. AMERICA & 36 & 1.9385 & 3.4053 & $(3)(4)(6)$ & \\
\hline (8) US & 61 & 2.6096 & 2.5807 & $(3)(6)$ & \\
\hline (7) S. AMERICA & 12 & 2.6469 & 1.3944 & $(3)(6)$ & \\
\hline (4) EUROPE & 49 & 4.2287 & 1.9927 & $(1)(2)(3)(5)(6)$ & III \\
\hline
\end{tabular}

Note. F $=22.366, p<0.001$.

\subsection{The Spread Patterns of COVID-19 in January 2021}

To identify countries/regions with the same spread pattern as Hebei Province in China, Figure 8 shows the three spread clusters in color (i.e., all DCCs equal zero in a yellow, mild epidemic in green, and second wave in red). The curved lines shown in Figure 8 are subject to the CC of CNIC between entities greater than 0.98 .
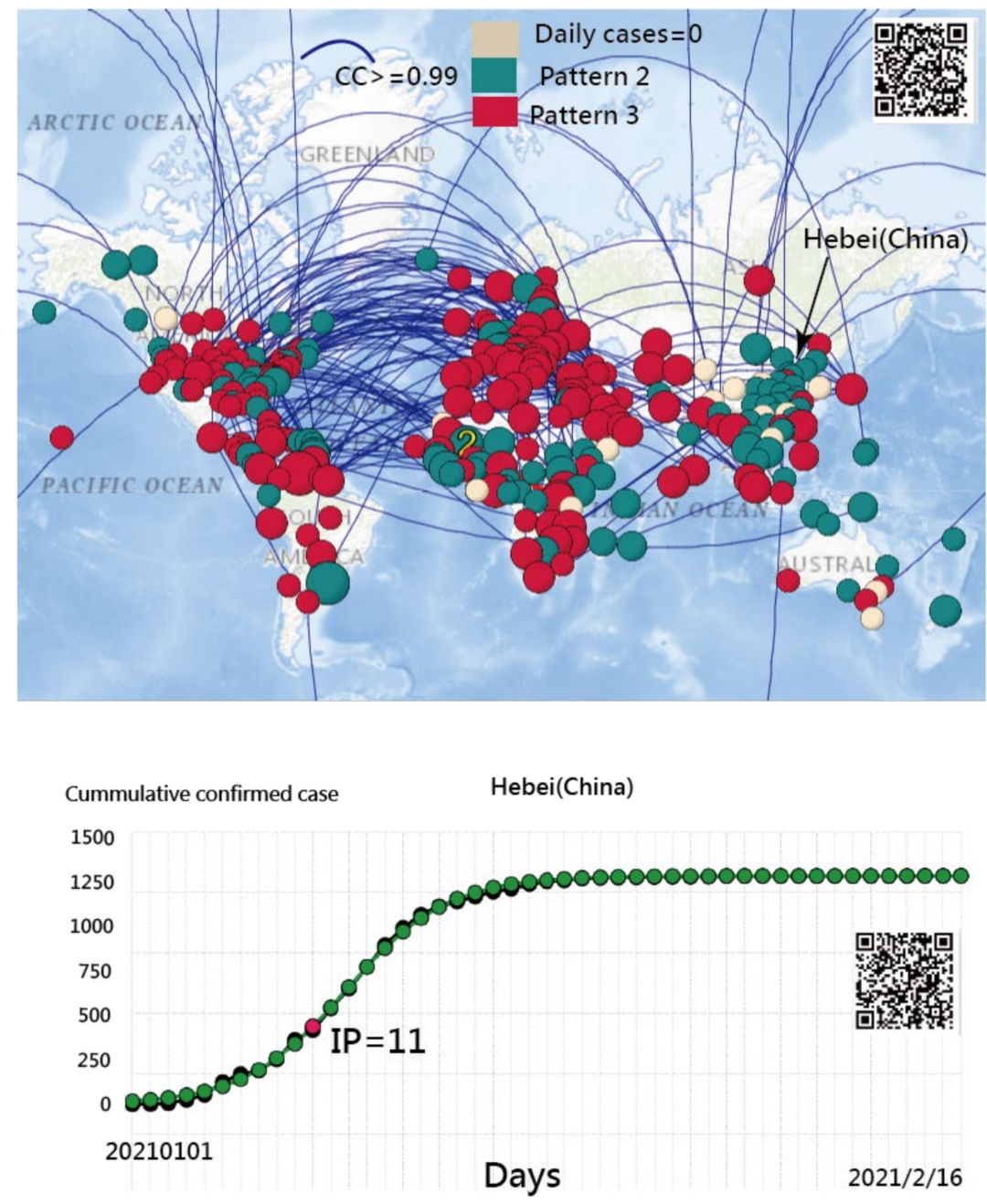

Figure 8. Spread patterns of COVID-19 in countries/regions using CCs ( $a=0.99, b=-2.48)$ (Note: readers are invited to scan the QR-code to examine the detail on a dashboard). 
Two findings related to the above phenomena were found: (1) Both the provinces of Hebei and Heilongjiang in China had a similar spread pattern in red bubbles; and (2) other countries (or regions), such as Japan, Taiwan, and Qatar, had the same spread pattern as Hebei Province (China) as well. Readers are invited to scan the QR code in Figure 8 and see the blue curved line linked to the bubble of Hebei (China). The country of Qatar is uniquely connected to Hebei (China). The comparison of IP days on the ogive curves is made for each country/region if the bubble is clicked on, such as the one for Hebei (China) shown at the bottom panel in Figure 8. The IRT model in MS Excel is shown in Appendix A.

\subsection{Online Dashboards Shown on Google Maps}

All those plots in Figures appear once the bubble or region of interest is clicked using the links, similar to those deposited in Appendix A.

\section{Discussion}

The classification has been used by humans for thousands of years. It is also important to our everyday life and applies to almost everything we do, allowing us to find and recognize things more easily. Imagine if we went to a library without classificationwhere would we start looking for a particular book? As of 18 January 2021, over 73,021 articles were found by searching the keyword "classification [title]" in Pubmed. Then, 57 articles were searched by "International journal of environmental research and public health [journal]," such as "machine learning-based activity pattern classification" [46], "support vector machine classification of drunk driving behavior" [47], and "local climate zone classification" [48], to name a few.

\subsection{Findings and Implications}

This study was classified by two periods during the COVID-19 pandemic: (1) From 22 January to 27 March 2020, and (2) from 1 January to 16 February 2021. We observed that (1) the use of SNA for investigating the SSP is viable; (2) the spread routes of COVID-19 from China to West Asia, Europe, North America, and South America are obvious in three classifications (255, $\mathrm{n}=51,130$, and 74 in stages); (3) those countries/regions of Heilongjiang (China), Japan, Taiwan, and Qatar are identical in a common cluster with Hebei Province (China), and (4) a dashboard on Google Maps can be applied to display the SSP of the COVID-19 around the world.

\subsection{What This Finding Adds to What We Already Knew}

Many online real- or near-real-time dashboards have been launched for tracking the worldwide spread of the COVID-19 outbreak [2-7]. Most of them are similar to other traditional websites [8-12], merely providing the same information as the WHO COVID-Dashboard [5]. The study of COVID-19 requires further mathematical analyses of data regarding cases and data (e.g., deaths and fatality rates) reported worldwide [49]. Although dashboards (e.g., JHU [2] and WHO [5], and others [3,4,6,7]) have provided interesting visualizations for reporting the current state of the COVID-19. However, these presentations lack important information and analysis approaches using mathematical models to predict the projection of DCC or CNIC, which would be useful to understand the trends of COVID-19 [49]. For instance, the COVID-19 Watcher [50] displays detailed COVID-19 data from every county and 188 metropolitan areas in the US. The drawback of it is also similar to other dashboards through different types of charts providing basically necessary information regarding COVID-19.

The COVID-19 Dashboard [49] was designed to offer additional valuable information (e.g., using a mathematical model to project future cases worldwide and by country). The mathematical model is based on linear algebra calculations with a quadratic equation using the last 31-day CNICs. Accordingly, the mathematical model [49] is able to estimate the number of new cases up to later several or more days. 
There have been criticisms of the UK government for not providing information on the evidence base used to inform their decision-making [51]. A real-time policy dashboard to aid global transparency in response to COVID-19 is expected to (1) present the localized reasoning behind COVID-19 policy decisions, and (2) allow the global health community to provide further support to governments and international stakeholders [52]. As such, dashboards only presenting real-time descriptions of new daily cases and risk factors are insufficient. A scientific approach involving the mathematical model embedded in the online dashboard is urgently required.

More than 36 articles of COVID-19 using SNA were published up to 16 February 2021 [53]. No authors have applied CCs of CNIC to explore the knowledge of spread patterns in a comparison among countries/regions. The SNA process is simple but useful, and defines the CCs between countries/regions prior to analysis. Two regions with a higher CC have a high probability within an identical cluster. Otherwise, these two will be in two distinctly different clusters [17-23]. The SSP was thus necessary to use SNA to classify attributes of COVID-19 for countries/regions (e.g., spread stages and occurrences of stationarity in the second wave during the COVID-19 pandemic).

\subsection{What Is Implied and What Should Be Changed}

The visual display, combined with SNA (e.g., in Figures 3 and 5), provides more messages to readers. This is the first study applying SNA to identify SSP of the COVID-19 cases around the world. Two types of SSP were included: (1) Spread stages from 22 January to 27 March 2020 and (2) occurrences of stationarity in the second wave from 1 January to 16 February 2021. These approaches are recommended to future studies and are not just limited to COVID-19 as in this study. The online dashboard involving SNA is as promising and viable as the online SNA illustrated on the website [54].

In addition to many mathematical models [24-31] used for predicting the CNIC in COVID-19, the IRT-based predictive model has been proposed [32]. The IP [13-16] on the ogive curve should be (and must be) determined before assessing the NISHC. The ogive curve [32] using Equation (5) to determine the IP and IPcase-index [32] evaluated the NISHC, which was different from previous studies $[55,56]$ which have used the DCC or CNIC alone.

The dashboard provided with SNA and IP on the given ogive curve is modern and innovative. Visual displays on CNIC using SSP were developed. We can see that red bubbles are in the UK and the US. The first confirmed case in the UK on 31 January 2020 [57] refers to the SSP in Figures 3 and 5, which is unique when compared to other COVID-19 dashboards $[3,4,58]$.

\subsection{Strengths of This Study}

The current study included three main stages: (1) SNA was used to classify the spread stages and occurrences of stationarity in the second wave during the COVID-19 crisis, (2) comparisons of model parameters (e.g., the location parameter or IP) were made to differentiate epidemic stages, and (3) an app was developed for understanding the transmission patterns of COVID-19 across countries/regions using the mathematical IRT model embedded in dashboards.

\subsection{Limitations and Future Studies}

Our study has some limitations. First, the CC was determined by NCIC between countries/regions. Further studies should compare whether the DCC is replaced by NCIC in the computation of CC.

Second, although we defined the CNIC as the proxy of the confirmed cases on continents to classify the spread patterns using SNA, we suggest that future research should include deaths to compare the difference in SSP.

Third, using the Microsoft Solver add-in to estimate IP days in the IRT model is not a unique approach. Many other methods can be applied to estimation, such as Warm's 
weighted mean likelihood estimate [59], anchored maximum likelihood estimation [60], and weighted likelihood estimation [61]. These methods are worthy of comparison in future studies.

Fourth, visual dashboards are shown on Google Maps. However, these installments are not free of charge. For example, the Google Maps application programming interface (API) requires a paid project key for the cloud platform. Thus, the limitations of the dashboard are that it is not publicly accessible, and it is difficult for other authors or programmers to mimic for use in a short period of time.

Fifth, although IRT is common and popular in the educational and psychometric field, many readers in public health are unfamiliar with IRT. The IRT 2-parameter model requires some effort to understand through the data and MP4 videos provided in the Appendix A.

Sixth, only two periods, (1) from 22 January to 27 March 2020 and (2) from 1 January to 16 February 2021, were included in this study. The spread pattern would be varied if different periods were observed because the SARS-CoV-2 virus mutates fast $[62,63]$.

Finally, the mathematical model was proposed in a previous study [49]. Further verification is required to determine whether the model residual is smaller than the IRT model [32]. The comparison study is easy to conduct if the study period is identical (e.g., 31 days [49]) in these two scenarios.

\section{Conclusions}

Two aspects, (1) the spread stages from 22 January to 27 March 2020, and (2) the occurrences of stationarity of second wave in January 2021, were observed using SNA techniques. An app was applied to display the cluster features during the COVID-19 pandemic. More details about the application are given in Appendix A with an MP4 video. An online dashboard developed for displaying the transmission patterns across countries/regions is recommended for future research.

Author Contributions: T.-W.C. developed the study concept and design. K.-Y.Y., Y.-T.Y. and W.C. analyzed and interpreted the data. S.-B.S. monitored the process of this study and helped in responding to the reviewers' advice and comments. T.-W.C. drafted the manuscript, and all authors provided critical revisions for important intellectual content. The study was supervised by S.-B.S. All authors have read and agreed to the published version of the manuscript.

Funding: This research received no external funding.

Institutional Review Board Statement: Not applicable for studies not involving humans.

Informed Consent Statement: Not applicable for studies not involving humans.

Data Availability Statement: All data were deposited in Appendix A.

Acknowledgments: We thank Enago (www.enago.tw (accessed on 20 February 2021)) for the English language revision.

Conflicts of Interest: The funders had no role in the design of the study; in the collection, analyses, or interpretation of data; in the writing of the manuscript, or in the decision to publish the results.

\section{Appendix A}

Appendix A: Resource of data, Excel modules, Pajek file, and MP4 videos involved in this study at https:/ / osf.io/nyga9/?view_only=8adda14da4a74f218a8bfc2b1cb3939e (accessed on 20 February 2021).

\section{References}

1. Feng, Y.; Li, Q.; Tong, X.; Wang, R.; Zhai, S.; Gao, C.; Lei, Z.; Chen, S.; Zhou, Y.; Wang, J.; et al. Spatiotemporal spread pattern of the COVID-19 cases in China. PLoS ONE 2020, 15, e0244351. [CrossRef]

2. Boulos, M.N.K.; Geraghty, E.M. Geographical tracking and mapping of coronavirus disease COVID-19/severe acute respiratory syndrome coronavirus 2 (SARS-CoV-2) epidemic and associated events around the world: How 21st century GIS technologies are supporting the global fight against outbreaks and epidemics. Int. J. Health Geogr. 2020, 19, 8. [CrossRef] 
3. JHU. Dashboard Online for COVID-19 in Near Real Time. Available online: https://coronavirus.jhu.edu/map.html (accessed on 20 February 2021).

4. Leszkiewicz, A. Dashboard Online for COVID-19 in Near Real Time. Available online: https://avatorl.org/covid-19/ (accessed on 20 February 2021).

5. World Health Organization. Novel Coronavirus (COVID-19) Situation (Public Dashboard). Available online: https:/ / covid19. who.int/ (accessed on 20 February 2021).

6. HealthMap. Novel Coronavirus 2019-nCoV (Interactive Map). Available online: https://healthmap.org/wuhan/ (accessed on 20 February 2021).

7. Schiffmann, A. A Creator of One COVID-19 Dashboard. Available online: https:/ / ncov2019.live/ data (accessed on 20 February 2021).

8. Google Team. 2019 Novel Coronavirus (nCoV) Data Repository. Available online: https://github.com/CSSEGISandData/2019 -nCoV (accessed on 20 February 2021).

9. World Health Organization (WHO). Novel Coronavirus (2019-nCoV) Outbreak. Available online: https://www.who.int/ emergencies/diseases/novel-coronavirus-2019/situation-reports (accessed on 20 February 2021).

10. Centers for Disease Control and Prevention (CDC). CDC Tests for 2019-nCoV. Available online: https://www.cdc.gov/ (accessed on 20 February 2021).

11. European Centre for Disease Prevention and Control (ECDC). Novel Coronavirus. Available online: https://www.ecdc.europa. eu/en/home (accessed on 20 February 2021).

12. DXY. Novel Coronavirus (2019-nCoV). Available online: https:/ /ncov.dxy.cn/ncovh5/view/pneumonia (accessed on 20 February 2021).

13. Duan, Q.; Wu, J.; Wu, G.; Wang, Y.G. Predication of Inflection Point and Outbreak Size of COVID-19 in New Epicentres. Nonlinear Dyn. 2020, 101, 1561-1581.

14. Chatham, W.W. Treating Covid-19 at the Inflection Point. J. Rheumatol. 2020, 47, 1-10.

15. Gu, C.; Zhu, J.; Sun, Y.; Ahou, K.; Gu, J. The inflection point about COVID-19 may have passed. Sci. Bull. 2020, 65, 865-867. [CrossRef]

16. Fan, R.G.; Wang, Y.B.; Luo, M.; Zhang, Y.Q.; Zhu, C.P. SEIR-Based COVID-19 Transmission Model and Inflection Point Prediction Analysis. J. Univ. Electron. Sci. Technol. China 2020, 49. [CrossRef]

17. Evelien, O.; Ronald, R. Social network analysis: A powerful strategy, also for the information sciences. J. Inf. Sci. 2002, 28, 441-453.

18. Martin, G. A social network analysis of Twitter: Mapping the digital humanities community. Cogent Arts Humanit. 2016, 3, 1171458. [CrossRef]

19. Hagen, L.; Neely, S.; Robert-Cooperman, C.; Keller, T.; DePaula, N. Crisis Communications in the Age of Social Media: A Network Analysis of Zika-Related Tweets. Soc. Sci. Comput. Rev. Soc. Sci. Comput. Rev. 2018, 36, 523-541. [CrossRef]

20. Hsieh, W.T.; Chien, T.W.; Kuo, S.C.; Lin, H.J. Whether productive authors using the national health insurance database also achieve higher individual research metrics: A bibliometric study. Medicine 2020, 99, e18631. [CrossRef]

21. Lin, C.H.; Chou, P.H.; Chou, W.; Chien, T.W. Using the Kano model to display the most cited authors and affiliated countries in schizophrenia research. Schizophr Res. 2019, 216, 422-428. [CrossRef]

22. Chien, T.W.; Wang, H.Y.; Chang, Y.; Kan, W.C. Using Google Maps to display the pattern of coauthor collaborations on the topic of schizophrenia: A systematic review between 1937 and 2017. Schizophr Res. 2019, 204, 206-213. [CrossRef]

23. Chien, T.W.; Chang, Y.; Wang, H.Y. Understanding the productive author who published papers in medicine using National Health Insurance Database: A systematic review and meta-analysis. Medicine 2018, 97, e9967. [CrossRef]

24. Perc, M.; Miksić, N.G.; Slavinec, M.; Stožer, A. Forecasting COVID-19. Front. Phys. 2020, 8, 127. [CrossRef]

25. Fang, Y.; Nie, Y.; Penny, M. Transmission dynamics of the COVID-19 outbreak and effectiveness of government interventions: A data-driven analysis. J. Med. Virol. 2020, 92, 645-659. [CrossRef]

26. Wu, J.T.; Leung, K.; Leung, G.M. Nowcasting and forecasting the potential domestic and international spread of the 2019-nCoV outbreak originating in Wuhan, China: A modelling study. Lancet 2020, 395, 689-697. [CrossRef]

27. Anastassopoulou, C.; Russo, L.; Tsakris, A.; Siettos, C. Data-based analysis, modeling and forecasting of the COVID-19 outbreak. PLoS ONE 2020, 15, e0230405. [CrossRef]

28. Zhao, S.; Chen, H. Modeling the epidemic dynamics and control of COVID-19 outbreak in China. Quant. Biol. 2020, 8, 11-19. [CrossRef]

29. Rong, X.; Yang, L.; Chu, H.; Fan, M. Effect of delay in diagnosis on transmission of COVID-19. Math. Biosci. Eng. 2020, 17, 2725-2740. [CrossRef]

30. Mandal, M.; Jana, S.; Nandi, S.K.; Khatua, A.; Adak, S.; Kar, T.K. A model based study on the dynamics of COVID-19: Prediction and control. Chaos Soliton Fract. 2020, 136, 109889. [CrossRef] [PubMed]

31. Huang, J.; Qi, G. Effects of control measures on the dynamics of COVID-19 and double-peak behavior in Spain. Nonlinear Dyn. 2020. [CrossRef]

32. Wang, L.-Y.; Chien, T.-W.; Chou, W. Using the Ipcase-Index with Inflection Points and the Corresponding Case Numbers to Identify the Impact Hit by COVID-19 in China: An Observation Study. Int. J. Environ. Res. Public Health 2021, 18, 1994. [CrossRef]

33. Lord, F.M. Practical applications of item characteristic curve theory. J. Educ. Meas. 1977, 14, 117-138. [CrossRef]

34. Lord, F.M. Applications of Item Response Theory to Practical Testing Problems; Lawrence Eribaum Associates: Hillsdale, NJ, USA, 1980. 
35. Huang, C.; Wang, Y.; Li, X.; Ren, L.; Zhao, J.; Hu, Y.; Zhang, L.; Fan, G.; Xu, J.; Gu, X.; et al. Clinical features of patients infected with 2019 novel coronavirus in Wuhan, China. Lancet 2020, 95, 497-506. [CrossRef]

36. CGTN News. COVID-19 in China: What's Happened in Hebei and Heilongjiang. Available online: https://news.cgtn.com/ news/2021-01-12/COVID-19-in-China-What-s-happened-in-Hebei-and-Heilongjiang-WZDgaNQeys/index.html (accessed on 12 January 2021).

37. CDC. Search COVID-19 Risk Assessment by Country. Available online: https://www.cdc.gov/coronavirus/2019-ncov/travelers/ index.html (accessed on 1 March 2020).

38. Batagelj, V.; Mrvar, A. Pajek-Analysis, and Visualization of Large Networks. In Graph Drawing Software; Jünger, M., Mutzel, P., Eds.; Springer: Berlin, Germany, 2003; pp. 77-103.

39. Chien, T.W. Cronbach's Alpha with the Dimension Coefficient to Jointly Assess a Scale's Quality. Rasch Meas. Trans. 2012, 26, 1379.

40. Lee, C.J.; Chou, W.; Chien, T.W.; Yeh, Y.T.; Jen, T.H. Using the separation index for identifying the dominant role in an organization: A case of publications in organization innovation. Int. J. Organ. Innov. 2020, 12, 135-145.

41. Chang, C.S.; Yeh, Y.T.; Chien, T.W.; Lin, J.C.J.; Cheng, B.W.; Lai, F.J. Using the separation index to identify the most dominant role: A case of application on COVID-19 outbreak. Int. J. Organ. Innov. 2020, 12, 10-20.

42. Kano, N.; Seraku, N.; Takahashi, F.; Tsuji, S. Attractive Quality and Must-Be Quality. J. Jpn. Soc. Qual. Control 1984, 41, 39-48.

43. Chou, P.H.; Yeh, Y.T.; Kan, W.C.; Chien, T.W.; Kuo, S.C. Using Kano diagrams to display the most cited article types, affiliated countries, authors and MeSH terms on spinal surgery in recent 12 years. Eur. J. Med. Res. 2021, 26, 22. [CrossRef] [PubMed]

44. Kan, W.C.; Chou, W.; Chien, T.W.; Yeh, Y.T.; Chou, P.H. The Most-Cited Authors Who Published Papers in JMIR mHealth and uHealth Using the Authorship-Weighted Scheme: Bibliometric Analysis. JMIR Mhealth Uhealth. 2020, 8, e11567. [CrossRef]

45. Chien, T.W. Comparison of Parameters in IRT Model for COVID-19 on the Kano Diagram. Available online: http://www. healthup.org.tw/gps/snapatternipoint.htm (accessed on 6 February 2021).

46. Park, J.; Kim, S. Machine Learning-Based Activity Pattern Classification Using Personal PM2.5 Exposure Information. Int. J. Environ. Res. Public Health 2020, 17, 6573. [CrossRef] [PubMed]

47. Chen, H.; Chen, L. Support Vector Machine Classification of Drunk Driving Behaviour. Int. J. Environ. Res. Public Health 2017, 14, 108. [CrossRef]

48. Quan, J. Multi-Temporal Effects of Urban Forms and Functions on Urban Heat Islands Based on Local Climate Zone Classification. Int. J. Environ. Res. Public Health 2019, 16, 2140. [CrossRef] [PubMed]

49. Florez, H.; Singh, S. Online dashboard and data analysis approach for assessing COVID-19 case and death data. F1000Research 2020, 9, 570. [CrossRef] [PubMed]

50. Wissel, B.D.; Van Camp, P.J.; Kouril, M.; Weis, C.; Glauser, T.A.; White, P.S.; Kohane, I.S.; Dexheimer, J.W. An interactive online dashboard for tracking COVID-19 in U.S. counties, cities, and states in real time. J. Am. Med. Inform. Assoc. 2020, 27, 1121-1125. [CrossRef]

51. Alwan, N.A.; Bhopal, R.; Burgess, R.A.; Colburn, T.; Cuevas, L.E.; Smith, G.D.; Egger, M.; Eldridge, S.; Gallo, V.; Gilthorpe, M.S.; et al. Evidence informing the UK's COVID-19 public health response must be transparent. Lancet 2020, 395, 1036-1037. [CrossRef]

52. THead, M.G. A real-time policy dashboard can aid global transparency in the response to coronavirus disease 2019. Int. Health 2020, 12, 373-374. [CrossRef]

53. Pubmed. Articles Related to COVID-19 and Social Network Analysis. Available online: https://pubmed.ncbi.nlm.nih.gov/ ?term $=\% 28 \% 28$ Social+network+analysis\%29+\%29+AND+\%28COVID-19\%5BMeSH+Major+T (accessed on 17 February 2021).

54. Gio, H.R. The Exploration of Author Network. Available online: https://researchoutput.ncku.edu.tw/en/persons/how-ranguo/network/ (accessed on 16 February 2021).

55. Chang, C.S.; Yeh, Y.T.; Chien, T.W.; Lin, J.J.; Cheng, B.W.; Kuo, S.C. The computation of case fatality rate for novel coronavirus (COVID-19) based on Bayes theorem: An observational study. Medicine 2020, 99, e19925. [CrossRef]

56. Majumder, M.S.; Rivers, C.; Lofgren, E.; Fisman, D. Estimation of MERS-Coronavirus Reproductive Number and Case Fatality Rate for the Spring 2014 Saudi Arabia Outbreak: Insights from Publicly Available Data. PLoS Curr. 2014, 6. [CrossRef]

57. Chien, T.W. The First Case in UK on 31 January 2020. Available online: http://www.healthup.org.tw / kpiall/wuhen2abc.asp? mid=UK\&mtype $=1 \& \operatorname{mlog}=1$ (accessed on 28 March 2020).

58. Schiffmann, A. nCoV2019.live. Available online: https:/ / ncov2019.live/data (accessed on 29 March 2020).

59. Linacre, J.M. The Efficacy of Warm's Weighted Mean Likelihood Estimate (WLE) Correction to Maximum Likelihood Estimate (MLE) Bias. Rasch Meas. Trans. 2009, 23, 1188-1189.

60. Linacre, J.M. Estimating Rasch measures with known polytomous (or rating scale) item difficulties: Anchored Maximum Likelihood Estimation (AMLE). Rasch Meas. Trans. 1998, 12, 638.

61. Warm, T.A. Weighted likelihood estimation of ability in item response theory. Psychometrik 1989, 54, 427-450. [CrossRef]

62. Mercatelli, D.; Giorgi, F.M. Geographic and Genomic Distribution of SARS-CoV-2 Mutations. Front. Microbiol. 2020, 11, 1800. [CrossRef] [PubMed]

63. Makarenkov, V.; Mazoure, B.; Rabusseau, G.; Legendre, P. Horizontal gene transfer and recombination analysis of SARS-CoV-2 genes helps discover its close relatives and shed light on its origin. BMC Ecol. Evol. 2021, 21, 5. [CrossRef] 